\title{
Role of diffusion weighted MRI in patients with hepatocellular carcinoma: prediction and
} assessment of response to trans-arterial chemoembolization

Rasha Mahmoud Gomaa ${ }^{a}$, Ahmed Hesham Said ${ }^{b}$ and Amal Gamal ${ }^{b}$

${ }^{a}$ Radiology department, Faculty of Medicine, Baniseuf University, Egypt

\begin{abstract}
:
Aim: The study goal is to detect if apparent diffusion coefficient (ADC), a quantitative parameter of diffusion-weighted imaging (DWI), can recognize HCC post TACE residuals. Results were obtained by using MRI_DWI \& ADC and were compared with those of Tri phasic-computed tomography (CT). patient and Methods M R I - DWI was performed to 20 patients with 24 HCC focal lesions before and 1month after TACE to calculate the ADC value of HCC. Patients were also evaluated with Tri phasic-CT after TACE. CT was performed within 1 month after TACE. Results: All patients under the study shows pretreatment restricted Diffusion weighted images with low ADC value. Mean ADC value before treatment was $1.14 \times 10^{-3} \pm 0.29 \times 10^{-3}$ and ranged from $0.819 \times 10^{-3}$ to $2.48 \times 10^{-3}$. 9 cases $(45 \%)$ cases show post-TACE good therapeutic response with facilitated diffusion \& mean $\mathrm{ADC}$ value $1.32 \times 10^{-3}$ with significant increase compared to pre-treatment values. 11 cases $(55 \%)$ shows post-TACE partial or no therapeutic response with restricted diffusion \& mean $\mathrm{ADC}$ value $1.18 \times 10^{-3}$. However, there was no statistical significant difference between $\mathrm{ADC}$ value of resolved and that of residual cases $(\mathrm{P}$-value $=0.067)$, DWI could predict the response of treatment as CT by $100 \%$. All cases of Good therapeutic response had a facilitated diffusion, all cases of partial therapeutic response had a restricted diffusion but less than pretreatment and all cases with no therapeutic response had a restricted diffusion but more than pretreatment. Conclusion DWI could evaluate HCC necrosis of tumor after chemoembolization, and the $\mathrm{ADC}$ importance might be its ability to detect viable necrotic tumor tissues. Furthermore, DWI-MRI determines improved liver lesion location. So, DWI can be used as an option for HCC patients short term follow up after chemoembolization and may direct patient control for decreasing radiation CT examination exposure and the danger of contrast materialinduced nephropathy.
\end{abstract}

Keywords : HCC-hepatic- malignant- TACE -DW MRI-ADC. 


\section{Introduction:}

The most widely recognized primary hepatic malignant cancer is Hepatocellular carcinoma (HCC) which is the fifth most prevalent malignant disease and the third most basic worldwide reason for deaths due to cancer. In 2000, there were 564,000 new cases and 549,000 mortality rates from HCC around the world, demonstrating the overwhelming evaluation of this tumour (1).

However using IFN as example in controlling chronic hepatitis $\mathrm{C}$ can reduce risk of hepatocellular carcinoma (2), HCV still one of major causes of hepatocellular carcinoma (3). In contrast to different types of malignant cancers, the $\mathrm{HCC}$ evaluation doesn't generally require histological affirmation and HCC is normally estimated by tumour marker and radiology like ultrasonography, C.T and X-ray (4).

Transplantation, trans-arterial treatments, different local ablative and liver resection are considered current efficacious therapy for HCC. Liver transplantation and liver resection are the primary therapeutic treatment. Unluckily, just about $20 \%$ patients, mostly investigated by screening regularly, may profit by these surgical techniques. (5).
The most common used therapy for HCC is chemoembolization, for cases not subjected to surgery. It depends on targeting of tumor de vascularization, wherein the oxygen and supplement supply to the tumor is blocked, leading to tumor death (6).

Likewise, TACE influences the tumor to the greatest effect of chemotherapy by specific or super-selective infusion of tumor vessels by chemotherapeutic agents which decrease blood flow to the tumor by particles embolization leading to delayed contact of the tumor with the chemotherapeutic agents. (7).

Liver magnetic resonance imaging (MRI) is gradually progressing from a critical solving imaging tool to a first line imaging methodology for lots of liver diseases. The MRI advantage over different cross sectional imaging tools might be the reason for this change. MRI technological advancements which emphasis on delivering great images and quick imaging, developing more recent function-specific contrast agents and enhancing accuracy of diagnosis are important in confirming that MRI is the most important diagnostic tool (8). 


\section{Patients and Methods:}

Our study protocol was approved by Bani Seuf University Research Ethics Review Committee. Written consent was obtained from all patients before starting the study.

\section{1 patient:}

Twenty patients were included (13 men \& 7 women: age ranged from 55 to 75 years, median age 65 year) with total 24 hepatic focal lesions (13 patient represents $65 \%$ have small nodules less than $5 \mathrm{~cm} \& 7$ patients represents $35 \%$ have large nodules more than $5 \mathrm{~cm}$ in diameters, 5 patients of them have infiltrative lesions). All diagnosed with HCC based on $\alpha$-fetoprotein levels and previous imaging findings \& are subjected for treatment with drug-eluting beads TACE.

MRI study was performed 1-2 days before TACE as well as 1month after. diffusion weighted images (DWI \& apparent diffusion coefficient (ADC) mapping were done. PostTACE Tri phasic-CT study done as well.

\subsection{MRI technique:}

MR imaging was performed on high field system (1.5 Tesla) magnet units (Philips
Achieva XR) utilizing a TORSO phased array coil.
a)
Diffusion study:

- DWI was performed utilizing respiratory stimulated fat-inhibited single-shot echo planar grouping that linked the two diffusion (motion probe) degrees around $180^{\circ}$ pulse along the three direction of segment select, frequency encoding, and phase encoding and information securing with an EPI readout was acquired by applying three various $b$ factors of 50,400, and $800 \mathrm{~s} / \mathrm{mm} 2$.

- Generalized auto- calibrating partially parallel acquisition (GRAPPA) imaging with a factor acceleration of two was applied to decrease the procurement time. Different parameters were as the following: repetition time $(\mathrm{TR}) \geq 1890 \mathrm{~m} \mathrm{sec}$, echo time $(\mathrm{TE})=70$ $\mathrm{m}$ sec, number of excitations $(\mathrm{NEX})=3$, matrix $124 \times 120$ with a field of view as little as possible, slice thickness $6.5 \mathrm{~mm}$, slice gap 1-2mm, scanning time 4-5 min. MR graphs were analyzed as follows:

- Signal intensity on diffusion with ADC values.

MR image interpretation:

DWI MRI:

1. Qualitative analysis: limited diffusion was recorded if the affected area has a bright 
sign on DWI that not reduced with higher $\mathrm{b}$ value and dark sign in the ADC map.

2. Quantitative analysis: (ADC estimation):- The mean ADC of every lesion recognized was estimated by applying (ROI) area of interest over the lesion.

\subsection{Tri phasic $-\mathrm{CT}$ :}

Tri phasic CT was done using a 64 slices Scanner (Toshiba Aquilion One) after injection of $1.3 \mathrm{ml} / \mathrm{kg}$ of iodinated contrast medium (Omnipaque $300 \mathrm{mg} / \mathrm{ml}$ ) using a 16G Needle in upper limb vein at rate of $4 \mathrm{ml} / \mathrm{sec}$ with following protocol:

1. arterial phase: acquired 20 seconds after peak calculated by a bolus tracking system with ROI in the abdominal aorta at a trigger density of 120 Hounsfield Units (HU);

2. portal phase acquired 70 seconds after contrast injection;

3. Delayed phase acquired 180 seconds after contrast injection.

\section{Inclusion criteria:}

HCC lesion cases subjected to perform drug eluting beads-TACE

- $\quad$ Males and females $>18$ years old.

- $\quad$ HCC (single nodule less than $8 \mathrm{~cm}$ or max. 3 nodules $\leq 3 \mathrm{~cm}$ ).
- $\quad$ Baseline CT or bone scan and MRI without radiological evidence perceptible major vascular intrusion or extra liver diseases.

- Hemoglobin $>9.0$ g/dl, WBC $>3000$ cells $/ \mathrm{mm}^{3}$.platelets $>75000$ cells $/ \mathrm{mm}^{3}$, bilirubin $<3 \mathrm{mg} / \mathrm{dl}$.

- $\quad$ Karnofsky list $>70 \%$

- $\quad$ Serum creatinine $<1.5 \mathrm{mg} / \mathrm{dl}$.

- $\quad$ INR/PTT $<1.5$ x maximum constraint of typical.

Patients with normal liver function (ChildPugh class $\mathrm{A} / \mathrm{B}$ ) without encephalopathy and mild to severe ascites

\section{Exclusion criteria:}

1. Contrast media contraindications, for example patients with kidney dysfunction, patients hypersensitive to contrast media.

2. Magnetic resonance imaging contraindications, for example claustrophobia in anesthesia contraindicated patients, non-MR compatible heart prosthesis, metallic plates, pace makes.

3. Hepatic cancers other than HCC. Patients who performed liver interventional techniques rather than transarterial chemoembolization (TACE) for example (RF, microwave ablation). 
All cases had been exposed to the following:

Full history taking:

Most patients are generally having symptoms of advanced cirrhosis like:

- $\quad$ Pruritus

- Jaundice

- $\quad$ Splenomegaly

- $\quad$ Variceal hemorrhage

- $\quad$ Cachexia

- Increasing abdominal dimension (occlusion of portal vein by thrombus with rapid ascites development)

Laboratory investigations revision of the patients including kidney and liver function tests. •

Patients were planned to perform MRI with DWI before and within one month after at DEB-TACE with estimating the value of $\mathrm{ADC}$.

All patients have triphasic-CT within onemonth post-TACE

We divide the patients into two groups:

- Good therapeutic response (resolved): shows complete iodine concentration with no enhancing tumoral tissue depending on Triphasic-CT study and correlated to MRI study (no MRI sins of areas of restricted diffusion).

- Partial therapeutic response (Unresolved): shows incomplete iodine concentration with residual enhancing tumoral tissue depending on the Triphasic-CT study and related to MRI DWI study if there is proof of residual tumor tissue with focal persistence of restricted diffusion.

- No therapeutic response (stationary or progressive): shows inadequate iodine concentration with residual enhancing tumoral tissue equal or larger than pretreatment tumor size depending on the Triphasic-CT study and related to MRI DWI study with persistence of restricted diffusion.

\section{Results:}

This study conducted on 20 patients complaining of HCC and underwent transarterial chemoembolization at tropical medicine department in Beni-Suef university hospital to investigate the role of diffusionweighted images in prediction and assessment of response to trans-arterial chemoembolization in cases of HCC. 
Table (1) Tumor characteristics of patients under the study:

\begin{tabular}{|c|c|c|}
\hline Tumor characteristics & Frequency & Percent \\
\hline $\begin{array}{l}\text { Stages of HCC } \\
-\mathrm{T} 1=\text { solitary lesion } 2 \mathrm{~cm} \text { without vascular } \\
\text { invasion } \\
-\mathrm{T} 2=\text { solitary lesion }>2 \mathrm{~cm} \text { with vascular } \\
\text { invasion or multiple tumors, none }>5 \mathrm{~cm} \\
-\mathrm{T} 3=\text { multiple }\end{array}$ & $\begin{array}{l}16 \\
3 \\
1\end{array}$ & $\begin{array}{l}80 \\
15 \\
\\
5\end{array}$ \\
\hline $\begin{array}{l}\text { Number } \\
\text {-Single } \\
\text {-Multiple }\end{array}$ & $\begin{array}{c}16 \\
4\end{array}$ & $\begin{array}{l}80 \\
20\end{array}$ \\
\hline $\begin{array}{l}\underline{\text { Size }} \\
- \text { Small }<5 \mathrm{~cm} \\
\text {-Large } \geq 5 \mathrm{~cm}\end{array}$ & $\begin{array}{c}13 \\
7\end{array}$ & $\begin{array}{l}65 \\
35\end{array}$ \\
\hline $\begin{array}{l}\text { Nodular/infiltrative } \\
\text {-Nodular } \\
\text {-Infiltrative }\end{array}$ & $\begin{array}{c}15 \\
5\end{array}$ & $\begin{array}{l}75 \\
25\end{array}$ \\
\hline $\begin{array}{l}\text { Pretreatment DWI } \\
\text {-Restricted }\end{array}$ & 20 & 100 \\
\hline
\end{tabular}

\section{Categorical data was presented as number and percent}

Table (1) showed that all patients under the study shows pretreatment restricted Diffusion weighted images with low ADC value (100\%). 


\section{Figure(2) CT results after treatment}

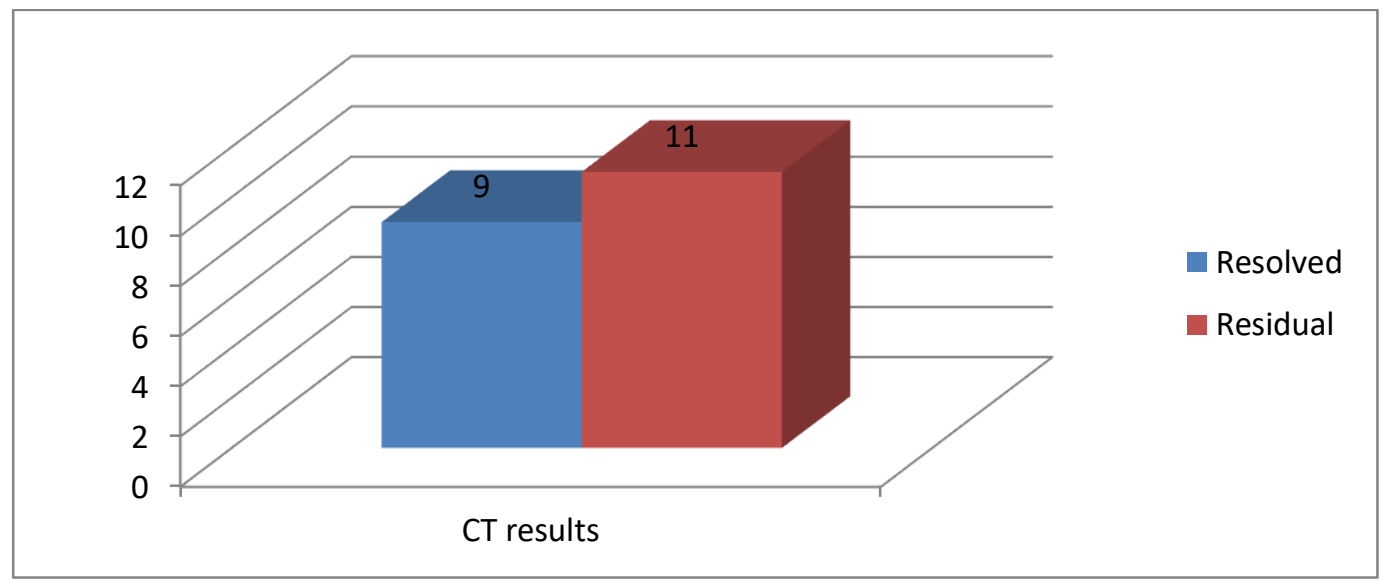

Figure (1) showed that $9(45 \%)$ cases with good therapeutic response after treatment and 11 $(55 \%)$ with partial or no therapeutic response.

Table (2) Diffusion weighted Image and ADC values after treatment

\begin{tabular}{|l|c|}
\hline characteristics & N(\%)/ Value \\
\hline Post-treatment DWI & $\underline{11(55)}$ \\
Festricted & $9(45)$ \\
\hline Post-treatment ADC value & \\
Mean & \\
SD & $1.24 \times 10^{-3}$ \\
Minimum & $0.25 \times 10^{-3}$ \\
Maximum & $0.858 \times 10^{-3}$ \\
Median & $1.777 \times 10^{-3}$ \\
& $1.22 \times 10^{-3}$ \\
\hline
\end{tabular}

\section{Categorical data was presented as number and percent}

\section{Scale data presented as mean and standard deviation (SD)}

Table (2) demonstrated that 11(55\%) cases of HCC had post-treatment restricted diffusion and 9 (45\%) had facilitated diffusion with mean post-treatment ADC value $1.24 \times 10^{-3} \pm 0.25 \times 10^{-3}$ ranged from $0.858 \times 10^{-3}$ to $1.777 \times 10^{-3}$ 
Table (3) comparison between the resolved and residual cases of $\mathrm{HCC}$ regarding the ADC value after treatment

\begin{tabular}{|l|c|c|c|}
\hline ADC value after ttt & Resolved & Residual & P-value \\
\hline Mean & & & \\
SD & 1.32 & 1.18 & \\
Minimum & 0.208 & 0.275 & \\
Maximum & 1.003 & 0.858 & \\
Median & 1.777 & 1.669 & \\
\hline
\end{tabular}

Scale data presented as mean and standard deviation $(S D) * P$-value is significant at $<0.05$ Table (3) showed that there was no statistical significant difference between ADC value of resolved and that of residual cases $(\mathrm{P}$-value $=0.067)$

Table (4) Agreement between CT results and DWI results after treatment:

\begin{tabular}{|c|c|c|c|c|}
\hline $\mathrm{CT}$ & Good & & & Total \\
\hline DWI & $\begin{array}{l}\text { therapeut } \\
\text { ic } \\
\text { response }\end{array}$ & $\begin{array}{c}\text { Partial } \\
\text { therapeutic } \\
\text { response }\end{array}$ & $\begin{array}{c}\text { No } \\
\text { therapeutic } \\
\text { response }\end{array}$ & \\
\hline Facilitated & $9(100 \%)$ & ------- & ------- & $9(45 \%)$ \\
\hline $\begin{array}{l}\text { restricted less than pre } \\
\text { treatment }\end{array}$ & ------ & $5(100 \%)$ & $\begin{array}{ll}---- \\
\end{array}$ & $5(25 \%)$ \\
\hline $\begin{array}{l}\text { restricted equal or } \\
\text { larger than pre } \\
\text { treatment }\end{array}$ & $\begin{array}{ll}------ \\
\end{array}$ & $\begin{array}{ll}---- \\
\end{array}$ & $6(100 \%)$ & $6(30 \%)$ \\
\hline Total & $9(100 \%)$ & $5(100 \%)$ & $6(100 \%)$ & $20(100 \%)$ \\
\hline
\end{tabular}


Table (4) showed that the DWI can predict the response of treatment as CT by $100 \%$. Also, this table illustrated that all cases of Good therapeutic response had a facilitated diffusion, all cases of partial therapeutic response had a restricted diffusion but less than pretreatment and all cases with no therapeutic response had a restricted diffusion but more than pretreatment.

Figure (2) Receiver operator characteristic curve: Prediction of resolving of HCC from ADC values after treatment:

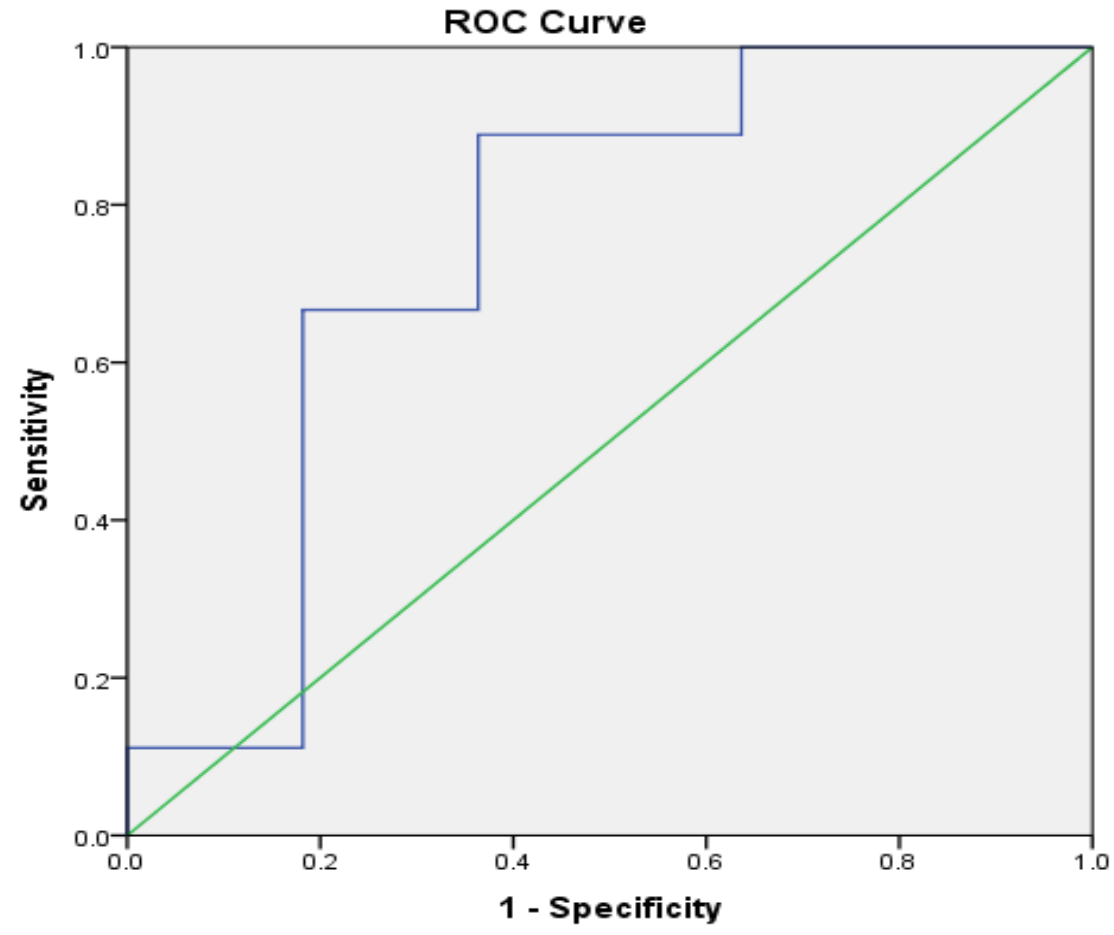

\begin{tabular}{|c|c|c|c|}
\hline \multicolumn{3}{|c|}{ Area Under the Curve } \\
\hline Test Result Variable(s): Post treatment ADC value \\
\hline Area & Std. Error & \multicolumn{2}{|c|}{ Asymptotic 95\% Confidence Interval } \\
\cline { 2 - 4 } & & Lower Bound & Upper Bound \\
\hline 0.747 & 0.115 & 0.522 & 0.973 \\
\hline
\end{tabular}

Figure (3) showed that the receiver operator characteristic curve for prediction of resolving HCC (by CT) scan be predicted from ADC value as follow:

At a cut off $1.19 \times 10^{-3}$ of ADC the resolving HCC (referenced by CT) can be predicted by $90 \%$ sensitivity and $64 \%$ specificity. 
Table (5) Rate of increase and rate of decrease of the ADC value after treatment of HCC

\begin{tabular}{|c|c|c|}
\hline Rate of change & Values & Number of cases $(n=20)$ \\
\hline Rate of increase & & $15(75 \%)$ All cases with increased \\
\hline Mean \pm Std. Deviation & $16.6 \% \pm 7.9 \%$ & ADC: \\
\hline Median & $15.5 \%$ & $* 9$ cases with Good therapeutic \\
\hline Minimum & $2.8 \%$ & response \\
\hline Maximum & $30.7 \%$ & $\begin{array}{l}* 6 \text { cases with partial therapeutic } \\
\text { response }\end{array}$ \\
\hline \multicolumn{3}{|l|}{ Rate of decrease } \\
\hline Mean \pm Std. Deviation & $-20.4 \% \pm 9.9 \%$ & $\mathbf{5}(\mathbf{2 5 \%})$ All cases with decreased \\
\hline Median & $-14.72 \%$ & ADC had residual of HCC (no \\
\hline Minimum & $-36.9 \%$ & therapeutic response) \\
\hline Maximum & $-13.3 \%$ & \\
\hline
\end{tabular}

Table (5) showed that there were 15 cases had an increase of ADC value after treatment with $16.6 \%, 9$ cases from them had radiological features of good therapeutic response with no gross residuals and 6 cases had partial response with residual viable tumor tissue. There were 5 cases of HCC after treatment had decreased ADC value and all of them had CT signs of residual of HCC with progression.

(a)

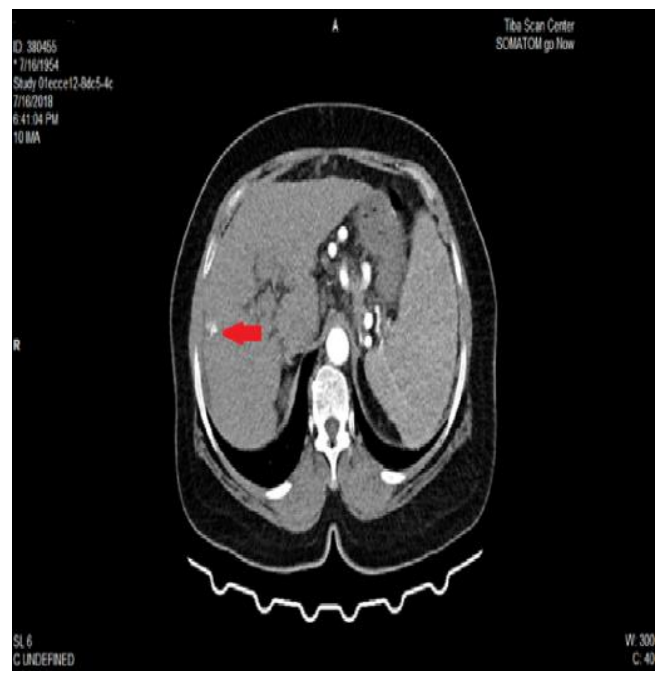

(b)

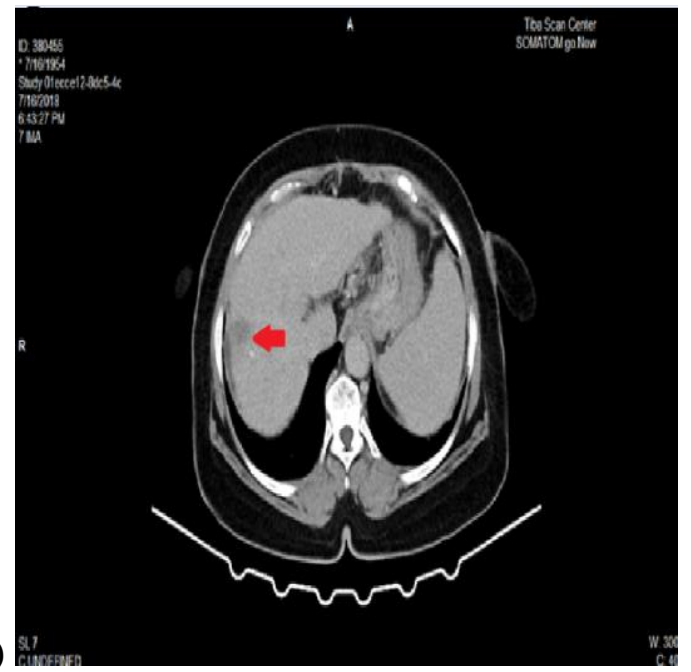

(figure: 3) female patient 62 years old underwent TACE. Triphasic-CT study axial cuts arterial phase and delayed phase (a)arterial phase (b)delayed phase, shows cirrhotic liver with ablated 10 
right hepatic lobe segment VII focal lesion. The lesion shows intra-lesional dense lipidol granules with no significant abnormal enhancement in arterial phase.
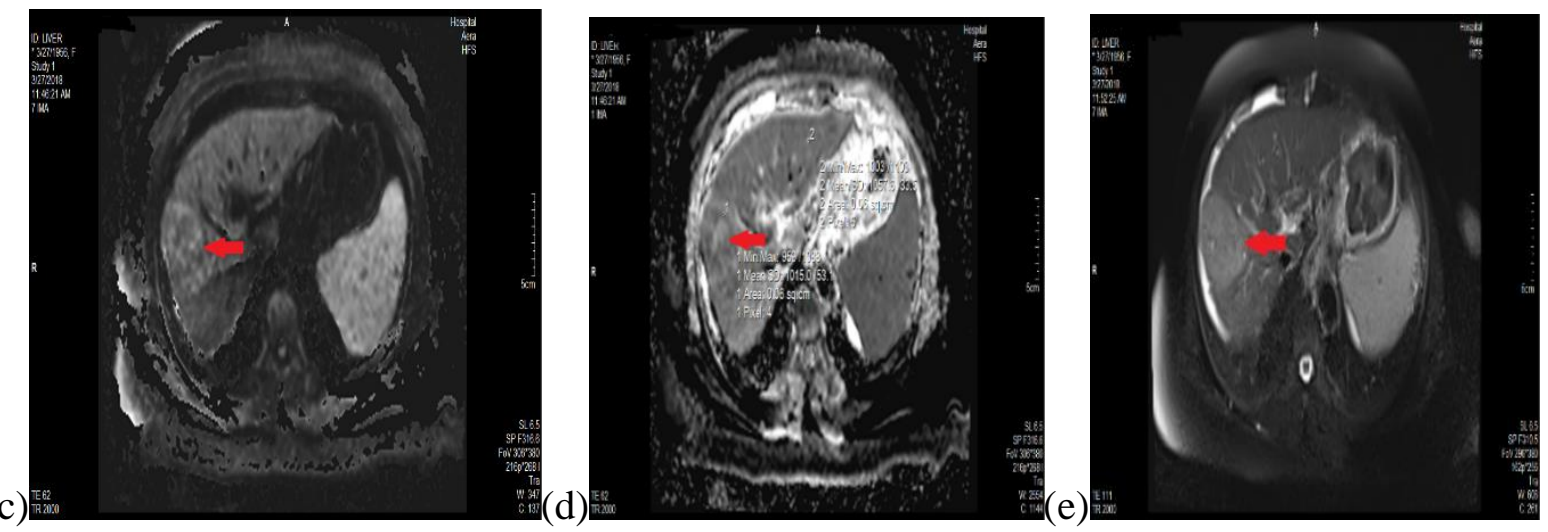

MRI study before TACE axial cuts; (c)Diffusion weighted image (d)ADC map \& (e) T2 weighted image before TACE. shows restricted diffusion of mass at segment VII with apparent diffusion coefficient value of $1.015 \times 10-3 \mathrm{~mm} 2 / \mathrm{sec}$.
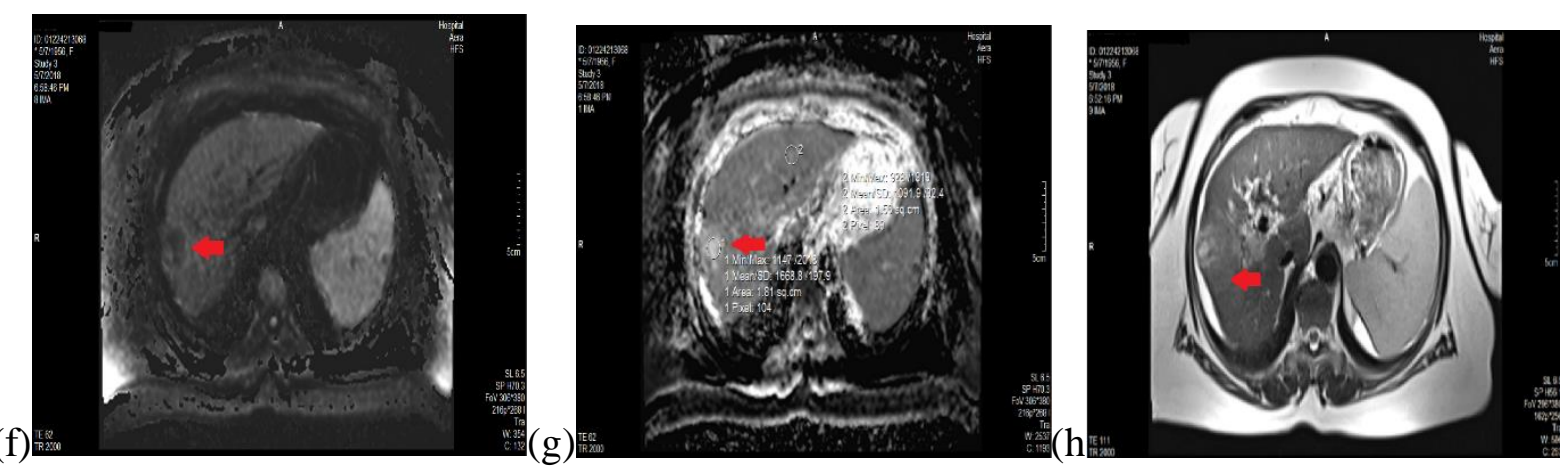

MRI study after TACE axial cuts DWI and ADC map(c)Diffusion weighted image(d)ADC map \& (h) T2 weighted image after TACE, shows mass with partially facilitated diffusion \& increased apparent diffusion coefficient value of $1.668 \times 10-3 \mathrm{~mm} 2 / \mathrm{sec}$. with reduced size, indicating good response. 

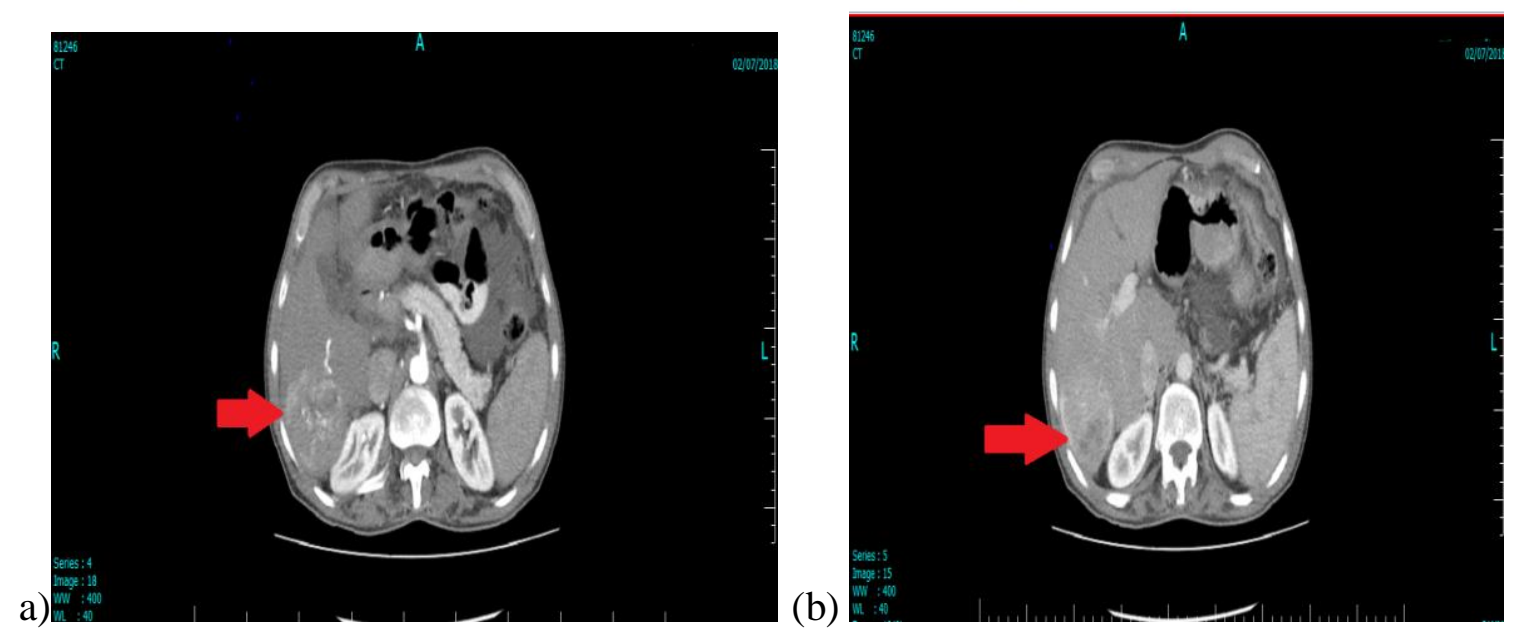

(figure: 4) male patient 62 years old; Triphasic-CT study after TACE axial cut arterial phase and portal phases. (a) arterial phase (b)portal phase shows cirrhotic liver with large well defined right hepatic lobe focal lesion, segment (VI). The lesion shows minimal accumulation of lipidol droplets with residual patchy areas of heterogeneous enhancement in the arterial phase and washout of contrast on the portal phase indicating residual tumoral activity.
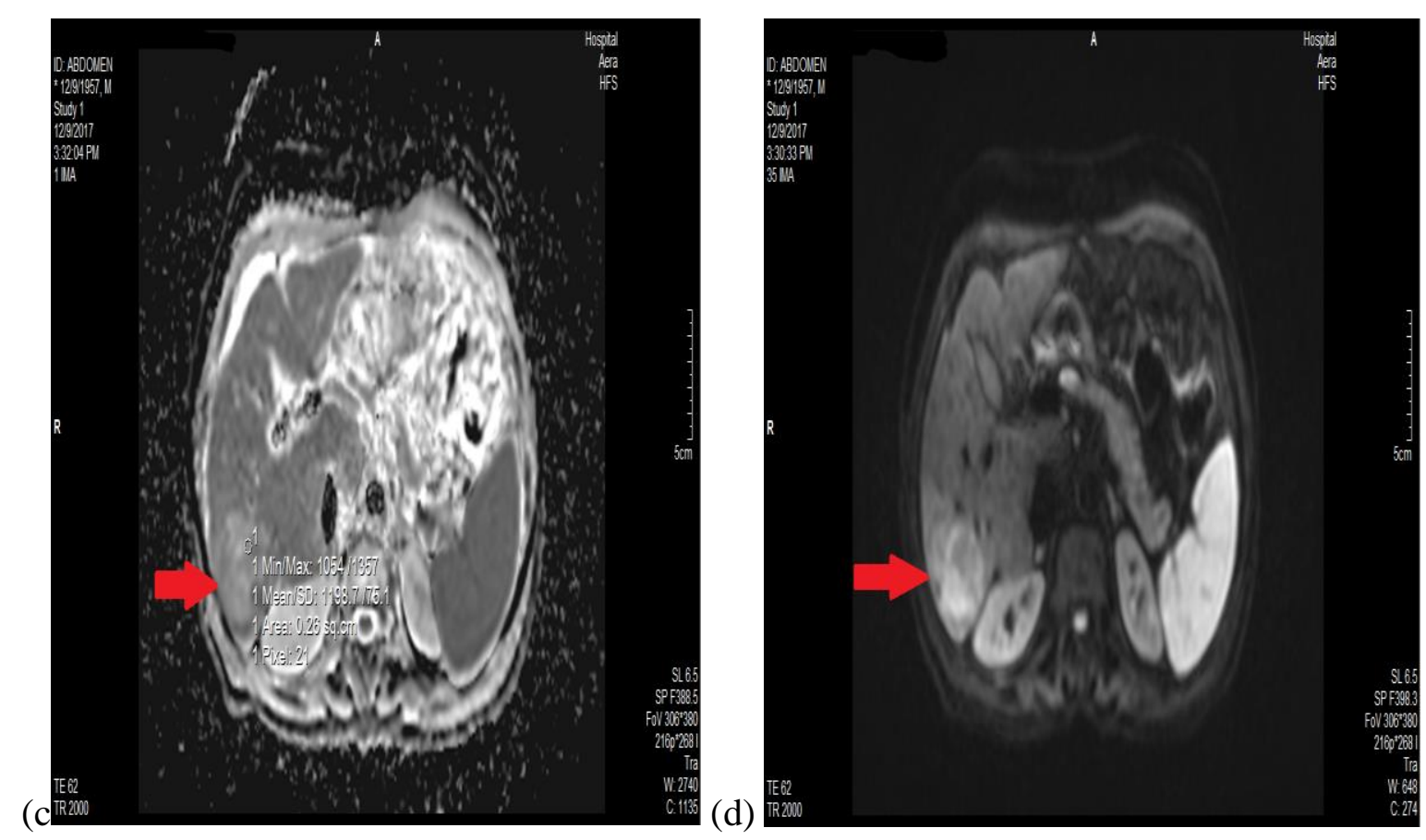

MRI study axial cuts before TACE; DWI and ADC map (a)ADC map (b)DWI, shows mass with restricted diffusion with apparent diffusion coefficient value of 1.19 x10-3 mm2/sec. 

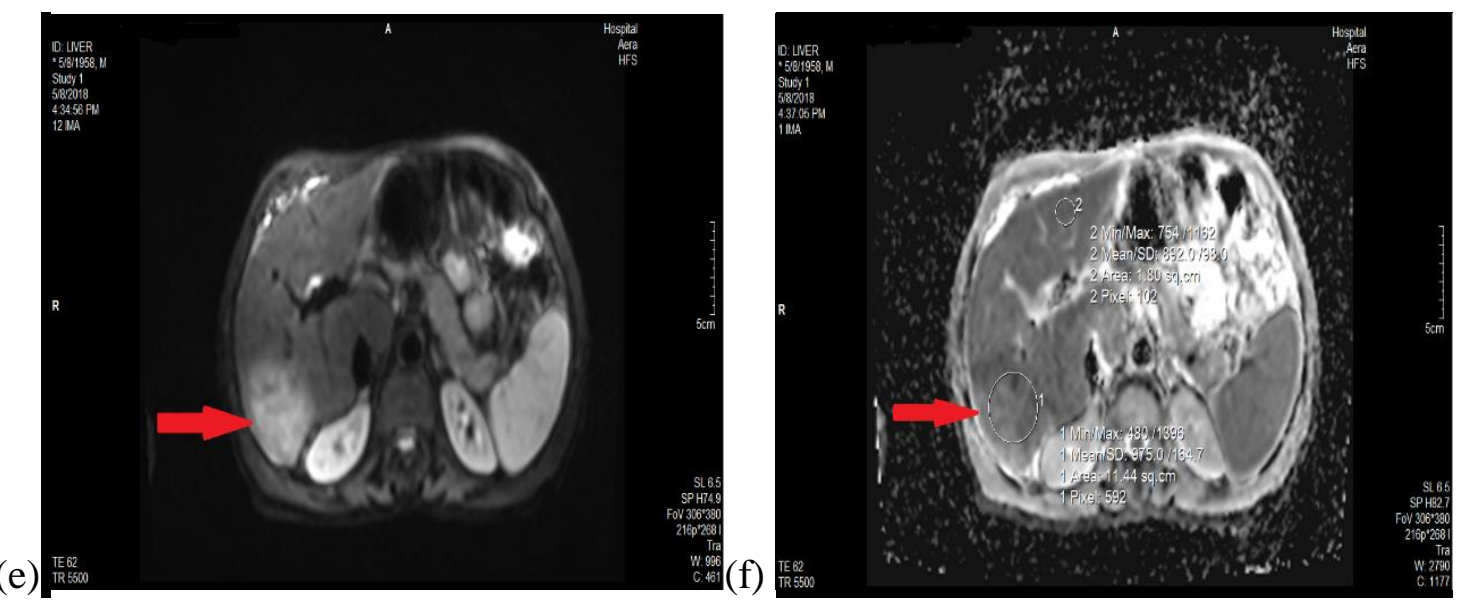

MRI study axial cuts post-TACE; DWI and ADC map (e)ADC map (f)DWI, shows mass still restricted diffusion with decreased apparent diffusion coefficient value of $0.975 \times 10-3 \mathrm{~mm} 2 / \mathrm{sec}$.

\section{Discussion:}

Hepatocellular carcinoma (HCC) is the $6^{\text {th }}$ most prevalent malignancy around the world with poor prognosis. (9). TriphasicCT widely used for post-TACE HCC assessment depending on presence or absence of contrast enhancement; however, high-attenuation lipidol can cause beamhardening artifact that may hind intralesional viable tumor tissue. (10).

DWI is a MRI sequence, which utilizes a specific imaging contrast system depends on the spread of water's atoms in the tissues. Contrasts in the limitation of water spread allow identification of typical and atypical tissue. (11).

Diffusion weighted Imaging (DWI) can be assessed quantitatively for apparent diffusion coefficient (ADC) assessment with 13 utilization of various b values (as example 0 , 500 and $1000 \mathrm{~s} / \mathrm{mm} 2)$. Diffusion weighted Imaging (DWI) gives specific data about tumor cell membrane integrity and cellularity and so, this way might be effective to show any changes in the micro environmental of tumor, which happen in response to therapy. It has been demonstrated that apparent diffusion coefficient has been increased significantly after trans-catheter chemo-embolization in hepatocellular carcinoma. (12).

The goal of the current study was to demonstrate the rule of DWI in recognition of presence of viable tumor tissue in hepatocellular carcinoma after TACE utilizing ADC value compared to Tri phasicCT. According to post-TACE, ADC values patients are classified in to good post 
therapeutic response, partial \& poor (no) therapeutic response. In cases with good post therapeutic response, DWI shows facilitation compared to pre-treatment scan with increased ADC values with mean ADC value before treatment was $\left(0.924 \times 10^{-3}\right)$ compared to $\left(1.32 \times 10^{-3}\right)$ with significant increase $(P=0.04)$. these results match results of previous studies provided that significant correlation between tumor response and percent change in $\mathrm{ADC}$ at days 4 and 39 post-treatment (13).

Kamel et al. also reported that lesional mean ADC value increased after TACE by 20\% $(P=0.026)$. (14) All our cases with good therapeutic response shows no significant tumoral tissue enhancement in post-TACE Triphasic CT with total (100\%) agreement with DWI/ADC results. In cases with partial therapeutic response, DWI still shows restricted diffusion yet with elevated ADC values compared to pretreatment values. In such cases residual enhancing tumoral tissue detected in post-TACE Triphasic-CT study. In cases with poor (no) therapeutic response, DWI still restricted diffusion with reduced ADC values compared to pretreatment values. In such cases residual enhancing tumoral tissue detected in post-TACE Triphasic-CT study.
We assess an ADC cut off value of HCC good response from the curve of ROC. We inferred that, 64\% specificity and $90 \%$ sensitivity can predict necrotic HCC. In this study, the necrotic lesions mean ADC value was $1.32 \times 10-3 \mathrm{~mm} 2 / \mathrm{sec}$.

The residual disease mean ADC value was $1.18 \times 10-3 \mathrm{~mm} 2 / \mathrm{sec}$ with no statistical difference $(\mathrm{P}=0.067)$. Compared to Lu T. et al., 2012, demonstrated that values of viable lesions were $0.97 \pm 0.39 \times 10-3 \mathrm{~mm} 2 / \mathrm{sec}$ and nonviable ones were $1.18 \pm 0.34 \times 10-3$ $\mathrm{mm} 2 / \mathrm{sec} \quad(\mathrm{p}=0.002)$. our study yielded specificity of $64 \%$ and sensitivity of $90 \%$, with $100 \%$ total agreement. These results corresponded to Osama R. et al 2013, who detected that DWI had a sensitivity $100 \%$ of $65.5 \%$ specificity, and $80 \%$ total agreement.

DWI had the following false negative results reasons:

HCC cases with initial facilitated diffusion \& relative high ADC value. ROI circular (interest region) utilized for ADC may contain false regions of dead nearby tissue. Lesions of subcapsular periphery contained peritoneal fat, this may increase the values of ADC. Finally, subcentimetric lesions aren't obviously observed (15).

DWI false positive results reasons: 
HCC lesion should be evaluated before and after treatment to demonstrate the increase in $\mathrm{ADC}$ percentage. ADC values may wrongly have lowered by necrosis and hemorrhage. Intra-lesional restriction may occur as a result to accumulation of Intralesional lipidol. Study limitation factors: Lack of follow up imaging and histopathologic assessment which the best to evaluate the normal tissue of tumor as well as relatively small sample size. To conclude,

\section{Summary:}

(DWI) gives specific data about tumor cell membrane integration and cellularity. Hence, DWI is precise to microenvironment changes that happen within tumor after therapy. This can be assessed quantitatively for the estimation of ADC. Specifically, the level of tumor necrosis of huge HCC after TACE might be recognized by DWI. So, DWI not only provides staying away from exposure to radiation during $\mathrm{CT}$ assessment and the danger of nephropathy, it also might be used as an instrument for short term follow up after TACE HCCs' directing further management of patient.

In our study each of the 20 TACE ablated HCCs' were analyzed according to DW-MRI. Lesions were classified according to morphological changes in size and
DWI could evaluate HCC necrosis of tumor after chemoembolization, and the ADC importance might be its ability to detect viable necrotic tumor tissues. Furthermore, DWI-MRI determines improved liver lesion location. So, DWI can be used as an option for HCC patients short term follow up after chemoembolization and may direct patient control for decreasing radiation CT examination exposure and the danger of contrast material-induced nephropathy.

enhancement on Tri phasic-CT study into resolved "good therapeutic response" group, "partial therapeutic response " group \& poor therapeutic response group. Furthermore the DWI was evaluated for areas of "facilitation" or "restriction" and ROI was drawn on those areas in the ADC maps. The ADC value obtained was recorded.

We found that DWI is a promising strategy which is very important in assessing HCC according to its ability to differentiate tissue histology. It makes it easy to distinct between necrotic and viable tumor regions and to help in residual tumor diagnosis.

The increase in diffusion coefficient can be used as a detector for potential treatment efficacy. After TACE follow up, there was 
significant increase in $\mathrm{ADC}$ values \& facilitated diffusion in good therapeutic response group compared to pre-treatment group. While in partial therapeutic response there was non-significant increase in ADC values with still residual restricted diffusion. In contrast to cases with poor therapeutic response there was reduces $\mathrm{ADC}$ value with restricted diffusion.

\section{References:}

1. Shariff, et al. Mohamed IF, "Hepatocellular carcinoma: current trends in worldwide epidemiology, risk factors, diagnosis and therapeutics." Expert review of gastroenterology \& hepatology 3.4 (2009): 353-367.

2. Mansour Hassan Ahmad Hassan, Dr. Mohamed Osman ${ }^{\mathrm{a}}$, Dr. Waleed ElNabawy $b$ and Dr. Alzahraa Yasseen Sayed, "Retinal Changes In Patients with Hepatitis C Virus under treatment with interferon and ribavirin" Egyptian Journal of Medical Research (EJMR), Volume 1, Issue 1, 2020.

3. Basel Abdelmonem Ebeid a, Alaa Aboud Muhammed a, Shaimaa Ali Abd Elkareem b and Asmaa Srour Soliman. "Predictive value \&changes in ChildPugh score in chronic hepatitis $\mathrm{C}$ cirrhotic patients treated with Direct
Acting Antiviral agents" Egyptian Journal of Medical Research (EJMR), Volume 1, Issue2, 2020

4. Bolondi, L., et al. Bolondi, L., et al. "Surveillance programme of cirrhotic patients for early diagnosis and treatment of hepatocellular carcinoma: a cost effectiveness analysis." Gut 48.2 (2001): 251-259.

5. Yau, Thomas, et al. "Evolution of systemic therapy of advanced hepatocellular carcinoma." World journal of gastroenterology: WJG 14.42 (2008): 6437.

6. Geschwind JF, Liapi, Eleni, and JeanFrancois. "Interventional oncology: new options for interstitial treatments and intravascular approaches: Targeting tumor metabolism via a loco-regional approach: a new therapy against liver cancer." Journal of Hepato-BiliaryPancreatic Sciences 17.4 (2010): 405406. Goodman ZD and Terracciano L. Tumor and tumor like lesions of the liver. In: Burt AD, Portman BC, Ferrell LD, eds. MacSween's Pathology of the liver. Churchill Livingstone Elsevier 2007; 761-814.

7. Reham M.Osama, et al. "Role of dynamic contrast-enhanced and diffusion weighted MRI in evaluation of necrosis of 
hepatocellular carcinoma after chemoembolization." The Egyptian Journal of Radiology and Nuclear Medicine 44.4 (2013): 737-746.

8. Kum Chol Ri1,2*, Chol Kim1,2, Sun Il Choe1,3, Ju Hyok So4 and Se Hyok O1 "The Klf6-related super enhancer regulates Klf6-SV2 expression mediated proliferation in human hepatoma (HepG2) cells" Beni-Suef University Journal of Basic and Applied Sciences volume 8, Article number: 10 (2019).

9. Santhi Maniam \& Janio Szklaruk..."Magnetic resonance imaging: Review of imaging technique and overview of liver imaging" World journal of radiology2.8 (2010):309.

10. Kim KW, Lee JM, Choi BI (2011) Assessment of the treatment response of HCC. Abdom Imaging. 36:300-314

11. Jill S., Gluskin, Jill S., et al. "Hepatocellular carcinoma and diffusion-weighted MRI: detection and evaluation of treatment response." Journal of Cancer 7.11 (2016): 1565.

12. Mannelli, Lorenzo, et al. "Advances in diffusion-weighted imaging." Radiologic Clinics 53.3 (2015): 569-581.

13. R.J. Theilmann, R. Borders, T.P. Trouard, et al.Changes in water mobility measured by 17 diffusion MRI predict response of metastatic breast cancer to chemotherapy .Neoplasia, 6 (2004), pp. 831-837

14. Kamel, Ihab R., et al. "Role of diffusionweighted imaging in estimating tumor necrosis after chemoembolization of hepatocellular carcinoma." American Journal of Roentgenology 181.3 (2003): 708-710.

15. Yuan, Shaochun, et al. "Amphioxus as a model for investigating evolution of the vertebrate immune system." Developmental \& Comparative Immunology 48.2 (2015): 297-305. 